\title{
The Eye of the Laboratory Mouse Remains Anatomically Adapted for Natural Conditions
}

\author{
Jonathan M. Shupe Deborah M. Kristan Steven N. Austad \\ Deborah L. Stenkamp \\ Department of Biological Sciences, University of Idaho, Moscow, Idaho, USA
}

\section{Key Words}

Mouse $\cdot$ Eye $\cdot$ Photoreceptor $\cdot$ Ganglion cell .

Evolution · Domestication · Vision · Mammal changes to the eye in any variable measured, supporting the continued use of this animal as a model for a naturally adapted visual system.

Copyright $(2006$ S. Karger AG, Basel

\begin{abstract}
Evolutionary effects of domestication have been demonstrated for several body systems, including the eye, and for several vertebrate species, including the mouse. Given the importance of the laboratory mouse to vision science, we wished to determine whether the anatomical and histological features of the eyes of laboratory mice are distinct from those of their naturally adapted, wild counterparts. We measured dimensions and masses of whole eyes and lenses from a wild population plus three inbred strains (C57BL/6J, NZB/BINJ, and DBA/1J) of the house house, Mus musculus, as well as wild and outbred laboratory-domesticated stock of the deer mouse, Peromyscus maniculatus. Histological preparations from these eyes were used to determine outer nuclear layer thickness, linear density of the ganglion cell layer, and for indirect immunofluorescence evaluation of cone opsin expression. For all of these traits, no statistically significant differences were found between any laboratory strain and its wild counterpart. The evolutionary effects of domestication of mice therefore do not include
\end{abstract}

\section{Introduction}

The laboratory mouse (Mus musculus) has been of tremendous experimental utility for understanding the physiology and genetics of health and disease in several systems, including the visual system. However, the strains commonly used for these studies are the result of at least a century of purposeful as well as inadvertent selective breeding and inbreeding [Silver, 1995]. In addition, under laboratory conditions where food and water are abundant and easily found, opportunities for physical activity limited, temperatures comfortable, and predators missing, selection for a range of traits important to survival in the wild will be relaxed. Not surprisingly then, compared to their wild ancestors laboratory-adapted mice rarely bite their handlers, grow rapidly from birth, mature early, and have frequent large litters [Miller et al., 2002]. Compared with wild mice, domesticated strains also exhibit reduced risk-avoidance behavior [Augustsson and Meyerson, 2004], exploratory behaviors [Fernandez et al.,

\section{KARGER}

(C) 2006 S. Karger AG, Basel

Fax +41613061234 E-Mail karger@karger.ch www.karger.com www.karger.com/bbe
Deborah L. Stenkamp

Department of Biological Sciences

University of Idaho

Moscow, ID 83844-3015 (USA)

Tel. +1 208885 8963, Fax +1 208885 7501, E-Mail dstenkam@uidaho.edu 
2004], strength and agility [Dohm et al., 1994], and have shorter lifespans [Miller et al., 2002]. Other genetic and physiological changes accompanying laboratory domestication may not be so obvious. For instance, laboratory mice have longer telomeres than wild mice [Hemann and Greider, 2000]. Many strains also exhibit early life hearing loss [Zheng et al., 1999], and do not synthesize melatonin in their pineal glands [Ebihara et al., 1986; Goto et al., 1989].

There is an interest in understanding the genetic and environmental variables that lead to visual system defects such as myopia [Zhou and Williams, 1999], glaucoma [Danias et al., 2003], and retinal degenerations [Farber and Tsang, 2003]. Although the use of laboratory mice has been productive in identifying some of these variables, it is possible that the eyes of this animal model have been affected by the selective pressures of domestication so as to no longer represent a naturally adapted visual system. Indeed, in a laboratory environment deterioration of vision would be expected to entail no reproductive cost so that mutations harmful to vision could spread by genetic drift. Thus spontaneous mutations leading to early life degeneration of retinal photoreceptors are fixed in a number of mouse strains [Chang et al., 2002]. Our goal was to investigate whether more subtle changes in laboratory mice had occurred compared with wild animals. In addition, we wished to place the visual system of laboratory mice into a useful evolutionary and experimental context by comparing specific anatomical features of their eyes with those of wild-trapped M. musculus.

Regression of the eyes and visual parts of the brain is a well-documented occurrence in species that become adapted to darkened environments. The visual system is metabolically costly to maintain; selection can favor the loss or change of visual structures when they are no longer serving an adaptive purpose [Cooper et al., 1993]. Alternatively, the genes regulating eye formation and function may become selectively neutral and accumulate mutations.

There is also evidence that both natural and artificial selective pressures influence the visual system in more modest but still functionally significant ways. For example, domestic dogs have fewer retinal ganglion cells than wolves, their wild counterparts, suggesting lower visual acuity [Peichl, 1992]. Ranched mink have smaller eyes with fewer retinal ganglion cells and cone photoreceptors than wild mink [Steffen, 2000; cited in Kruska, 2005]. Although not yet explicitly demonstrated for changes to the eye, other evolutionary changes to the nervous system have been documented to take place over very short time frames [reviewed by Kruska, 2005]. For example, a 20\% reduction in brain volume in ranched vs. wild mink was evident following approximately 120 generations of captive breeding [Kruska, 1996]. Therefore it is reasonable to hypothesize that 50-300 generations of domestication in M. musculus [Silver, 1995] may be sufficient to result in changes to the visual system. However, domestication appears to have its greatest effect on nervous system structure and function in the more highly encephalized mammals (species with larger brains relative to their body size). Studies of brain mass have shown 20 to $34 \%$ decreases for domestic carnivores and artiodactylae as compared to their wild counterparts, but only 0 to $13 \%$ decreases are evident for domestic lagomorphs and rodents [reviewed by Kruska, 2005]. Interestingly, M. musculus is currently the only known example of no observed decrease in brain mass with domestication [Frick and Nord, 1963; cited in Kruska, 2005]. It is therefore reasonable to hypothesize that domestication of $M$. musculus may have resulted in no measurable changes elsewhere in the nervous system, such as in the eye. Finally, it is conceivable that the laboratory environment itself rather than any genetic changes due to domestication may have resulted in alterations of eye anatomy.

We have observed that the eyes of wild mice superficially appear larger than those of domesticated strains [Austad, 2002]. In the present study we measure eye size, lens size, density of cells in the outer nuclear layer and ganglion cell layer, and the density of cone photoreceptors in three commonly used strains of laboratory $M$. musculus and in locally obtained wild $M$. musculus. These measurements provide an indirect assessment of the size of the visual field, refractive capacities, light sensitivity, visual acuity and color vision. Because some of these parameters are known to change with age and with body mass, we performed statistical analyses to account for these variables. In order to look at a second possible case where laboratory domestication may have affected visual acuity, we separately evaluated a laboratory strain of Peromyscus maniculatus and locally obtained wild P. maniculatus for the same parameters. Peromyscus is apparently more reliant on nocturnal vision than Mus as suggested by the fact that their eyes are substantially larger.

For all analyses, we find no statistically significant difference between wild animals and domesticated stocks and strains. The visual system anatomy of domesticated $M$. musculus and $P$. maniculatus was not significantly different from that of their wild counterparts for any of the 
traits we measured. This study justifies the ongoing use of laboratory strains of $M$. musculus as valid models for a naturally adapted visual system.

\section{Materials and Methods}

\section{Animals}

Eyes were taken from 39 wild $M$. musculus and from nine each of the following laboratory Mus strains: C57BL/6J, NZB/BINJ, and $\mathrm{DBA} / 1 \mathrm{~J}$. These laboratory strains have no known retinal degeneration mutations. C57BL6J and DBA1J represent two of the oldest domesticated mouse varieties, having descended from 'fancy' show mice and then brother-sister inbred for approximately 200 generations [Silver, 1995]. NZB/BINJ has been brother-sister inbred since 1948 from original New Zealand Black domesticated parents [Silver, 1995]. We similarly sampled eight wild Peromyscus maniculatus and compared them with 13 individuals from a randomly bred laboratory stock descended from 40 individuals originally trapped near Ann Arbor, Michigan in 1948. The laboratory P. maniculatus have not been purposely inbred, although any captive colony of finite size will experience some degree of inbreeding over time. Our wild $M$. musculus and $P$. maniculatus of unknown age were trapped at a local lambing barn in Moscow, Idaho. In some cases, wildtrapped mice were bred in the laboratory and their $\mathrm{F}_{2}$ progeny were examined at known ages $(\mathrm{n}=27)$. All laboratory Mus strains were of known age and obtained from Jackson Laboratory (Bar Harbor, Me., USA) and Simonsen laboratories (Gilroy, Calif., USA). Wild Mus correspond to M. musculus musculus (Jackson Labs). P. maniculus bairdii were obtained from Peromyscus Genetic Stock Center (Columbia, S.C., USA). Wild Peromyscus were likely P. maniculatus sonoriensis or $P$. maniculatis bairdii, as these are the types found in Idaho [J Sullivan, personal communication, 2005]. Mice from both sexes were used in the study. Procedures for trapping, care, breeding, and sacrifice of mice were approved by the University of Idaho Animal Care and Use Committee and conformed with the ARVO Statement for the Use of Animals in Ophthalmic and Vision Research.

\section{Eye and Lens Measurements and Tissue Processing}

Mice were sacrificed by asphyxiation with carbon dioxide and whole body masses were measured. Eyes were immediately removed by cutting extra-ocular tissues and the optic nerve, and whole eye masses were measured. Axial and equatorial dimensions of the eyes were measured with calipers. Corneas were then pierced with a microscalpel and entire eyes were placed in a buffered, $4 \%$ paraformaldehyde solution for $1-2 \mathrm{~h}$ and then re-examined for the effects of fixation by measuring eye mass and axial and equatorial lengths. Lenses were removed and weighed and measured in the axial and equatorial directions. The fixed eyes (without lenses) were placed in a buffered, $5 \%$ sucrose solution for $30 \mathrm{~min}$.

Left eyes were radially incised from the cornea in the eyecup towards the optic nerve. Retinas were carefully removed with curved forceps and placed in a $50 \% \mathrm{MeOH}$ solution for $5 \mathrm{~min}$ and then stored as whole mounts in $100 \% \mathrm{MeOH}$ at $-20^{\circ} \mathrm{C}$.

Right eyes were washed in progressively increasing concentrations of buffered sucrose $(10,12.5,15 \%)$, and then placed in a $20 \%$ sucrose solution overnight at $-20^{\circ} \mathrm{C}$. These cryoprotected eyes were washed in a 2:1 20\% sucrose solution/OCT medium for $30 \mathrm{~min}$ and embedded in this medium for cryosectioning. Sections were cut at $5 \mu \mathrm{m}$ and mounted with 4',6-diamidino-2-phenylindole (DAPI) medium to identify individual cells within the photoreceptor cell layer (outer nuclear layer; ONL) and the ganglion cell layer (GCL) using fluorescent microscopy.

\section{Immunocytochemistry}

Rabbit polyclonal antibodies directed against mouse M-opsin and mouse S-opsin were purchased from Chemicon (Temecula, Calif., USA); immunocytochemistry was performed as previously described [Stenkamp et al., 2000; Stenkamp and Frey, 2003]. In brief, following a 30-minute (cryosections) or 1-hour(whole mounts) blocking step in $20 \%$ goat serum, antibodies were applied singly or together at $1: 250$ overnight at $4^{\circ} \mathrm{C}$ in $1 \%$ goat serum. Tissue was washed in phosphate-buffered saline containing $0.05 \%$ Triton X100 (PBST), then a Cy3-conjugated anti-rabbit antibody (Jackson Immunoresearch, Westgrove, Pa., USA) was applied at 1:200 for $2 \mathrm{~h}$ (cryosections) or overnight (whole mounts). Tissue was washed in PBST and mounted in carbonate-buffered $60 \%$ glycerol with $0.4 \%$ phenylenediamine to preserve fluorescence.

\section{Cell Counts}

Tissues were viewed on a Leica DMR compound microscope under epifluorescence optics, and images were collected using a Spot digital camera (Diagnostic Instruments, Sterling Heights, Mich., USA). In some cases, contrast was enhanced, using Adobe Photoshop software (Adobe Systems, Mountain View, Calif., USA), to facilitate identification and counting of DAPI-labeled nuclei.

Cells were counted from digital pictures taken only from sections that ran through the optic nerve head to ensure that the cross section was representative of the entire eye. Our goal was to obtain data for comparative purposes within this study, not absolute numbers. However, in the interest of collecting data that could more readily be compared to similar data in the literature, the following steps were used: (a) to avoid counting portions of cells, only DAPIlabeled nuclei were counted; and (b) to avoid double-counting cells, counts were done from sections collected at least $15 \mu \mathrm{m}$ apart from each other. To compare the number of cells of the photoreceptor layers of different mouse strains, the number of rows of nuclei in the ONL was counted from three different areas (one central, two peripheral) of each of three representative sections. To compare the number of cells of the GCLs of different mouse strains, the number of nuclei in this layer was counted over a photographic field, then linear density was calculated (number of GCL cells counted, divided by the curvilinear retinal distance evaluated - typically $100 \mu \mathrm{m})$. Linear densities were measured from three different areas (one central, two peripheral) of each of three representative sections.

To quantify cone photoreceptor labeling, the number of labeled cones was counted from two to five different rectangular areas (each typically $100-200 \mu \mathrm{m}$ on a side) of whole mounted retinal tissue, and an average density calculated. During processing, retinal orientation (i.e dorsal vs. ventral) was not maintained; therefore, to minimize the effects of topographic asymmetries of cone subtypes [Applebury et al., 2000], we analyzed regions from central retina, where these asymmetries are less evident.

\section{Statistics}

ANOVAs or t-tests, performed by SPSS software, were used to statistically evaluate cellular and morphological differences be- 
tween the wild mice and the various lab strains. Regression analyses were performed with MS Excel software. When appropriate, data are presented as mean \pm standard error.

\section{Results}

\section{Eye and Lens Mass}

Histological processing resulted in a loss of $20-25 \%$ of the original mass of the eye, comprising a loss of three to six $\mathrm{mg}$ for $M$. musculus and $12-15 \mathrm{mg}$ for $P$. maniculatus. This effect of fixation is far greater than the $6 \%$ loss in mass using the methods of Zhou and Williams [1999]; however, the extent of tissue shrinkage was highly consistent from individual to individual. Original and processed eye mass were highly correlated $(\mathrm{r}=0.991$; data not shown $)$ for both Mus and Peromyscus. In addition, our fixation method permitted further histological and immunocytochemical analysis. All measurements are shown as the average of the post-fix masses of right and left eyes. Measurements obtained from contralateral eyes were almost identical, having a correlation coefficient of 0.994 and were not statistically different in a paired t-test.

In $M$. musculus, average eye mass varied from 20.02 $\pm 3.15 \mathrm{mg}$ (wild) to $20.63 \pm 2.91 \mathrm{mg}$ (NZB/BINJ), and average lens mass varied from $4.6 \pm 0.49 \mathrm{mg}(\mathrm{DBA} / 1 \mathrm{~J})$ to $4.91 \pm 1.72 \mathrm{mg}$ (wild). An ANOVA revealed that eye and lens masses were not significantly different between the wild caught $M$. musculus and any of the three inbred strains: $\mathrm{F}_{3,50}=0.146, \mathrm{p}=0.932$ for the eye, and $\mathrm{F}_{3,50}=$ $0.118, p=0.949$ for the lens (fig. 1A, B). The same conclusion was drawn from the comparison between wild Peromyscus with the laboratory strain: $\mathrm{F}_{1,19}=0.001, \mathrm{p}=$ 0.973 for the eye, and $F_{1,19}=0.159, p=0.694$ for the lens (fig. 1A, B). The slightly greater variance of eye and lens mass for the wild $M$. musculus prompted us to evaluate left vs. right asymmetries; we found no significant differences in asymmetry among the Mus strains for either eye mass $(p=0.77)$ or lens mass $(p=0.73)$.

Because some of our experimental animals were wildtrapped, they could not be age-matched to their laboratory counterparts. Eye and lens size increase almost logarithmically for a multitude of inbred laboratory strains from 30 to 300 days of age [Zhou and Williams, 1999]. Therefore, it was possible that the unknown ages of our wild mice were influencing the results. We performed a regression analysis for individual mice of all strains, in all of the cases where ages were known. This analysis confirmed that eye mass increases as a function of the log of the age in the total population of Mus examined (fig. 1C), according to the following relationship: eye mass $(\mathrm{mg})=$ $2.8+10.2$ ( $\log$ of age). Although our data representing older, wild-derived (i.e., lab-reared $\mathrm{F}_{2}$ offspring of wild animals) Mus are limited, the distribution of eye mass as a function of age strongly resembles that found by Zhou and Williams [1999] for multiple laboratory strains. Similarly, lens mass and the mass of the eye-minus-lens follows the logarithmic increase in age and can be described by the equations: lens mass $(\mathrm{mg})=3.8(\log$ of age $)-1.7$, and eye-lens mass $(\mathrm{mg})=4.6+6.4$ (log of age) (fig. $1 \mathrm{D}$, E). These data again bear a remarkable resemblance to those of Zhou and Williams [1999], suggesting that age may influence eye and lens mass of wild mice in a manner similar to the situation in laboratory mice. We were unable to perform a similar analysis for the $P$. maniculatus data, because all wild $P$. maniculatus were wildtrapped and ages were not known.

Wild and wild-derived mice are physically smaller than age-matched laboratory counterparts [Miller et al., 2002]. We therefore pursued the relationships between body size and eye and lens size in the different mouse strains (fig. 1F, G). Body mass and eye and lens masses are positively correlated $(r=0.76$ and $r=0.62)$ with changes of $0.80 \mathrm{mg}$ in eye mass, and $0.47 \mathrm{mg}$ in lens mass for every 1-gram increase in body mass for M. musculus. There is some strain-specific variation in this relationship; the lenses of wild Mus show a slightly greater increase in mass for every 1-gram increase in body mass, but this difference is significant only if compared to pooled data from all laboratory strains. For P. maniculatus, the relationships between body size and eye and lens size are not highly correlated.

It is possible that the laboratory environment influences development and/or growth of the eyes, and that we might therefore have obtained misleading information regarding differences in eye and lens size when we evaluated the progeny of wild-trapped mice. To determine if

Fig. 1. Eye and lens masses for wild and laboratory strains of $M$. musculus and P. maniculatus. A Box plot of eye masses for wild and laboratory strains (box stretches from the 25 th percentile to the 75 th percentile; the median is a line across the box; box lies between minimum and maximum values). B Box plot of lens masses for wild and laboratory strains. C-E Regression analysis of eye mass (C), lens mass (D), and eye mass minus lens mass (E) as a function of the log of age (although actual age is indicated on the figure); each symbol represents data collected from a single mouse. All data shown are from $M$. musculus strains. F, G Regression analysis of eye mass (F) and lens mass (G) as a function of body mass for wild and laboratory $M$. musculus strains. 


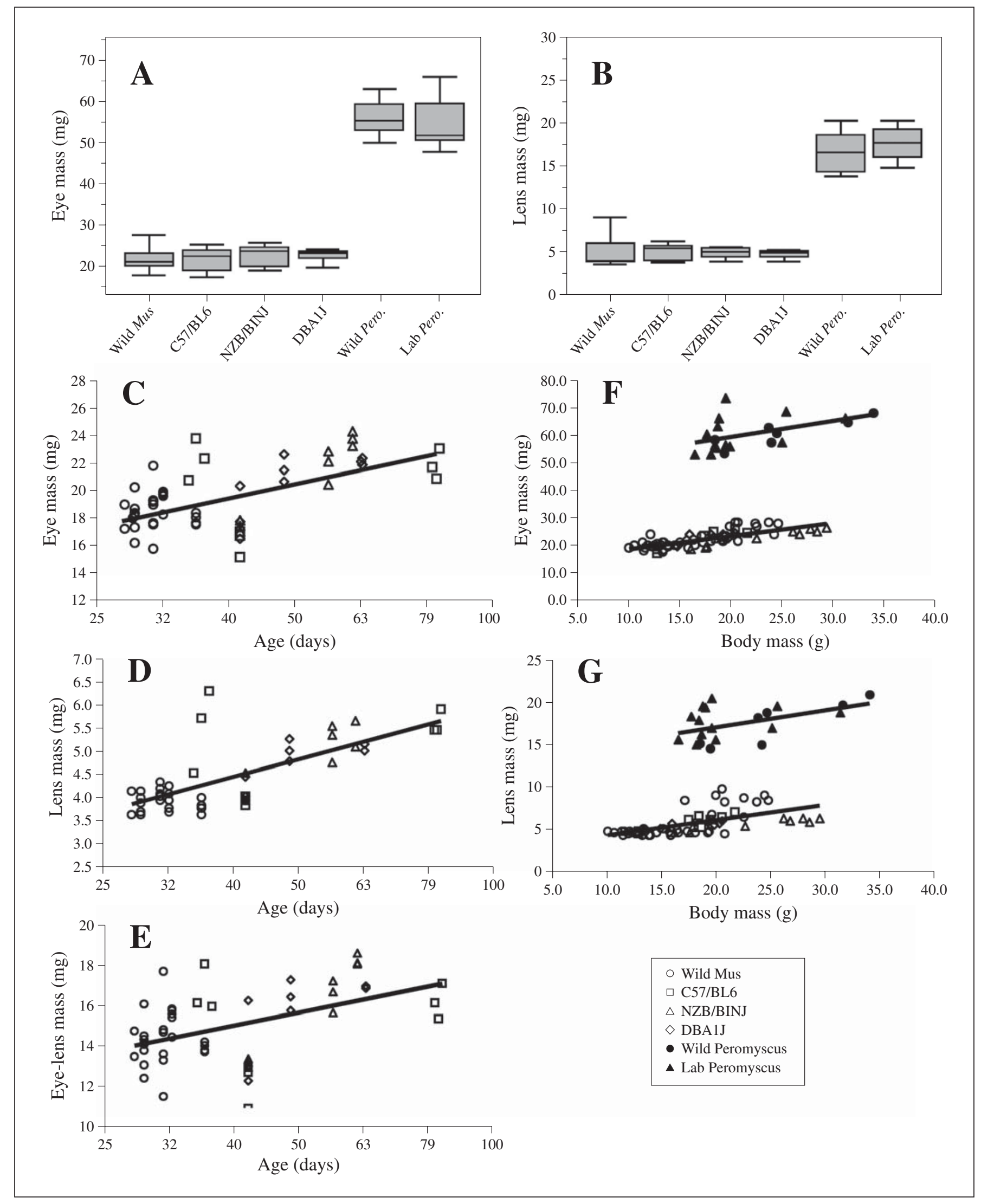




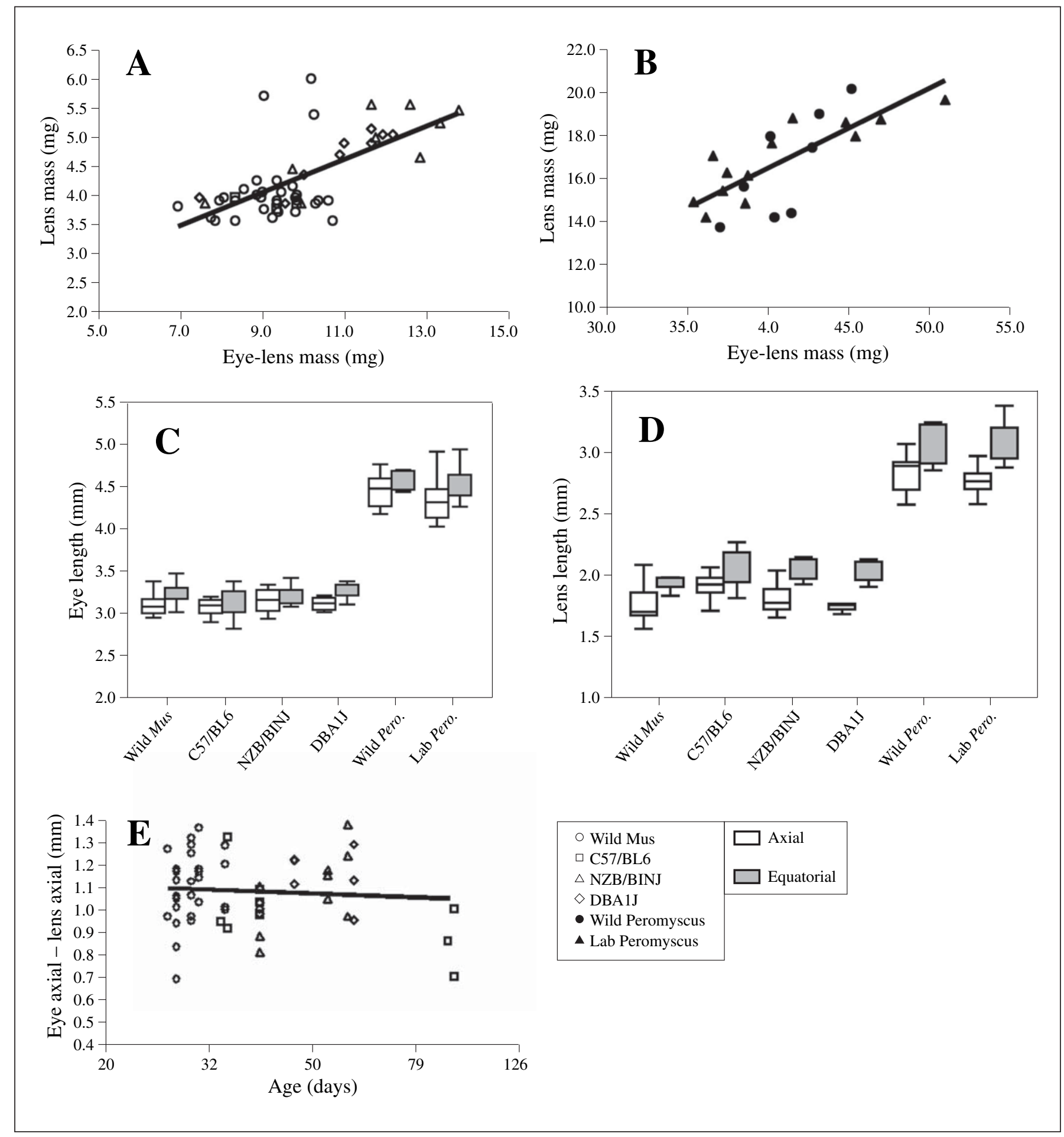

Fig. 2. Size relationships between eyes and lenses for wild and laboratory strains of $M$. musculus and P. maniculatus. A, B Regression analysis of lens mass as a function of the mass of the eye excluding the lens; each symbol represents data collected from a single mouse. A Wild and laboratory strains of M. musculus. B Wild and laboratory $P$. maniculatus. C Box plots of eye lengths in the axial and equatorial dimensions for wild and laboratory strains of
M. musculus and P. maniculatus. D Box plot of lens lengths in the axial and equatorial dimensions for wild and laboratory strains of M. musculus and P. maniculatus. E Regression analysis of eye axial length excluding the axial length of the lens (approximation of cornea thickness plus depth of the vitreous chamber) as a function of the log of age (although actual age is indicated on the figure); each symbol represents data collected from a single mouse. 
this was the case, we compared eye and lens mass in wildtrapped $M$. musculus to that of their mature lab-reared $\mathrm{F}_{2}$ progeny. We evaluated only mice that could be matched for body size, because eye mass varies as a function of both age and body mass [Zhou and Williams, 1999; and see above], and the ages of the wild-trapped mice were not known. There were no significant differences (eye mass, $p=0.61$; lens mass, $p=0.32$ ), indicating that the laboratory environment probably does not influence development and/or growth of the eyes of wild mice.

\section{Optical Parameters}

The capacity of the eye to properly focus images on the retina is in part a function of size of the eye and refractive properties of the lens and cornea. To indirectly assess and compare this capacity, we analyzed eye mass vs. the mass of the eye excluding the lens (fig. 2A). These parameters are highly positively correlated $(\mathrm{r}=0.97)$ with an increase of $0.73 \mathrm{mg}$ in lens weight for each 1-mg increase in eyelens weight. This correlation is stronger than that obtained by Zhou and Williams [1999], with less scatter about the line of best fit, perhaps because only four mouse strains were assessed in the present study, as compared to over 30 in the earlier study. A one-way ANOVA revealed no statistical difference between the wild mice and the three laboratory inbred strains $\left(\mathrm{F}_{3,50}=0.63 ; \mathrm{p}=0.60\right)$. A similar relationship between lens mass and eye-lens mass was obtained for Peromyscus.

Axial lengths of the eyes of Mus genotypes were similar, with a one-way ANOVA indicating no statistical difference between the wild mice and the three laboratory strains $\left(F_{3,60}=0.67 ; p=0.58\right.$; fig. $\left.2 \mathrm{C}\right)$; equatorial lengths also showed no significant differences $\left(\mathrm{F}_{3,60}=0.80 ; \mathrm{p}=\right.$ 0.50; fig. 2C). Dimensions of lenses also did not differ significantly by strain within species (fig. 2D). We again evaluated right vs. left asymmetry and found no significant differences for eye axial length $(p=0.71)$, eye equatorial length $(p=0.60)$, lens axial length $(p=0.48)$ and lens equatorial length $(\mathrm{p}=0.78)$ among the $M$. musculus strains.

Although both whole eye and lens size are positively correlated with age and with body mass [the present study; Zhou and Williams, 1999], the respective growth rates are not matched so as to retain a constant depth of the vitreous chamber [Schmucker and Schaeffel, 2004]. To indirectly assess whether this was the case with all strains sampled, we analyzed the difference between axial length of the eye and axial length of the lens (= vitreous chamber depth plus corneal thickness) as a function of age (fig. 2E). This measure showed a slight downward trend, similar to that of Schmucker and Schaeffel [2004], but with only a weak and nonsignificant correlation with age (linear relationship, $r=0.116$; log relationship, $r=0.084$ ).

\section{Retinal Histology}

Sectioned retinal tissue of wild $M$. musculus and $P$. maniculatus was superficially indistinguishable from that of laboratory Mus and Peromyscus, respectively (fig. 3A, B; data not shown for Peromyscus). All retinal layers were easily identified and similar in apparent thickness and cell number.

The average thickness of the ONL in Mus ranged from $9.73 \pm 0.37$ rows of nuclei in DBA/ $1 \mathrm{~J}$ to $10.58 \pm 1.01$ rows of nuclei in NZB/BINJ (fig. 3C). These values are similar to those of several strains routinely examined by others [e.g., Smith, 1992; Frederick et al., 2001], verifying that our counting techniques were reliable. No significant differences were found in the number of cells comprising the thickness of the ONL in the retina between the wild caught mice and the three laboratory strains $\left(\mathrm{F}_{3,10}=\right.$ $0.630 ; p=0.614$; d.f. $=14$ ). Similarly, ONL thickness in the two Peromyscus groups examined did not differ significantly (fig. 3C).

Linear densities of the GCL ranged from $87.1 \pm 4.5$ cells $/ \mathrm{mm}$ in the wild mice to $95.0 \pm 1.9$ cells $/ \mathrm{mm}$ in the NZB/BINJ strain (fig. 3C). The linear density of cells in the GCL of wild mice was not significantly different from that of any of the three laboratory strains examined $\left(F_{3,19}=0.659, p=0.588\right.$; d.f. $\left.=23\right)$. To estimate approximate planar densities (number of cells per $\mathrm{mm}^{2}$ of retina) and total number of GCL cells per retina, we squared the linear densities, with the assumption that our linear measurements provided sufficient topographic representation (see Methods). These estimated cell densities ranged from $7,710 \pm 838$ cells per $\mathrm{mm}^{2}$ (wild M. musculus) to $9,036 \pm 361$ cells per $\mathrm{mm}^{2}$ (NZB/BINJ). Approximately $60 \%$ of the nonvascular cells within the GCL of the laboratory mouse are ganglion cells [Dräger and Olsen, 1981; Jeon et al., 1998]. Therefore, the densities of actual ganglion cells in the mice strains examined are likely much lower, approximately 4,626 \pm 503 cells per $\mathrm{mm}^{2}$ (wild caught Mus) to $5,421 \pm 216$ cells per $\mathrm{mm}^{2}$ (DBA/1J), although without information regarding the ratio of ganglion cells to non ganglion cells in the GCL of wild mice this number remains speculative. Cell density in the GCL of Peromyscus was lower than that measured for Mus, with average linear densities of approximately 35 cells/ mm (fig. 3D). However, GCL cell densities of wild vs. laboratory Peromyscus strains did not statistically differ. 

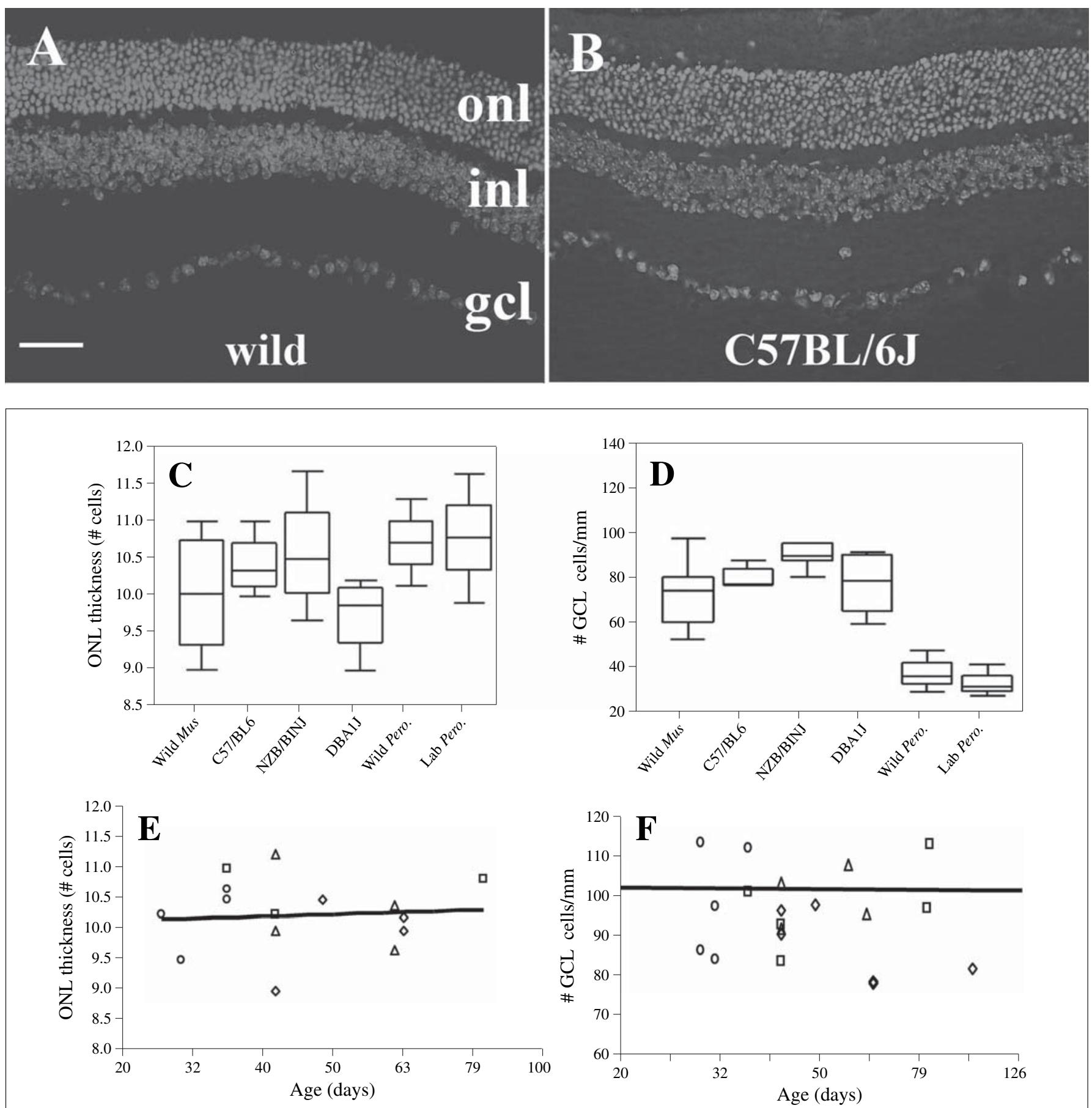

$\begin{array}{ll}\circ \text { Wild Mus } & \triangle \text { NZB/BINJ } \\ \square \text { C57/BL6 } & \diamond \text { DBA1J }\end{array}$

Fig. 3. Retinal histology and retinal cell numbers in wild and laboratory strains of $M$. musculus and $P$. maniculatus. A DAPI-labeled retinal cryosection obtained from a wild-trapped $M$. musculus. Bar $($ applies to A and B) $=50 \mu \mathrm{m}$. B DAPI-labeled retinal cryosection obtained from a C57BL/6J mouse; onl, outer nuclear layer; inl, inner nuclear layer; gcl, ganglion cell layer. C Box plots representing numbers of rows of nuclei in the ONL for all wild and laboratory strains. D Box plots representing linear density of cells in the GCL for all wild and laboratory strains. E Regression analysis of ONL thickness as a function of the log of age for wild and laboratory strains of M. musculus; each symbol represents data collected from a single mouse. F Regression analysis of cell density in the GCL as a function of the log of age (although actual age is indicated in the figure) for wild and laboratory strains of M. musculus. 
As with our eye size measurements, some of our data were obtained from wild mice of unknown age, others from laboratory-reared descendents of wild-trapped mice. There is evidence for both developmental- and aging-related change in both ganglion cell and photoreceptor number in several vertebrates [Gao and Hollyfield, 1992; Kunert et al., 1999; Obin et al., 2000]. Therefore, our results could have been affected in an uncontrolled manner by our use of animals of unknown age. We evaluated ONL thickness and GCL linear density as a function of age, for individual mice of all groups, in all of the cases where ages were known. This analysis revealed no change in ONL thickness as a function of age $(r=0.156$ for age; $r=0.049$ for log age; fig. 3E). A similar analysis revealed a negligible decrease in GCL cell density as a function of age, again with very weak correlations with either age $(\mathrm{r}=$ $0.046)$ or log age $(r=0.382$; fig. $3 F)$. We may not have evaluated any animals old enough to display age-related declines in photoreceptor or ganglion cell number. When animals of unknown age were excluded from this analysis, we found no statistically significant differences among groups for either ONL thickness or ganglion cell density (data not shown).

\section{Cone Photoreceptors}

Antibodies directed against S- and M-opsin selectively labeled cone outer segments in the four mouse strains used for this part of the study: C57BL/6J Mus (35 and 42 days old), wild Mus (32 and 36 days old), laboratory $P$. maniculatus (118 days old), and wild $P$. maniculatus (unknown age; fig. 4A-D for Mus; fig. 4L-O for Peromyscus). To compare the relative densities of each cone phenotype, we performed whole mount immunocytochemistry using each antibody (fig. 4E-H). Densities of S-opsin-expressing cones in the two Mus strains examined did not statistically differ $(\mathrm{p}=0.775)$; similarly, the density of M-opsin-expressing cones in wild $M$. musculus did not differ significantly from that in C57BL/6J mice $(p=0.3608)$. The number of M-opsin-expressing cones in each strain was similar to the number of S-opsin-expressing cones, consistent with previous quantitative data [Applebury et al., 2000].

There is considerable evidence that most of the cone photoreceptors in the $\mathrm{DBA} / 1 \mathrm{~J}$ and in the $\mathrm{C} 57 \mathrm{BL} / 6 \mathrm{~J}$ mouse strains express a combination of S- and M-opsin: all cones express M-opsin, although the levels of M-opsin are highest in dorsal and lowest in ventral retina; all but the most dorsal cones express S-opsin [Applebury et al., 2000]. Because we did not retain spatial orientation of the retina throughout our experiments, our cone density measure- ments were taken from central retina in order to minimize the effects of topographic gradients. To determine the extent to which wild vs. C57BL/6J mouse cones co-expressed S- and M-opsin, we performed immunocytochemical experiments using a combination of the two antibodies (fig. 4I-K). In these experiments, a slightly but significantly greater number of cones were labeled by this combination than by either antibody alone (fig. $4 \mathrm{~K}$ ). This was true for both Mus groups examined. The estimated total density of cone photoreceptors $\left(11,313 \pm 400\right.$ cones $/ \mathrm{mm}^{2}$, wild; $10,718 \pm 523$ cones $\left./ \mathrm{mm}^{2}, \mathrm{C} 57 \mathrm{BL} / 6\right)$ was similar to that reported previously using alternative methods and C57BL/6J mice $\left[12,000 / \mathrm{mm}^{2}\right.$ - Applebury et al., 2000; $12,400 / \mathrm{mm}^{2}-$ Jeon et al., 1998]. These findings are consistent with co-expression of two opsin genes in the cone photoreceptors of wild mice, suggesting that co-expression of opsins in the laboratory mouse is not the result of reduced evolutionary pressure for the maintenance of dichromacy. Our data also suggest that some cones may not express M-opsin, in contrast to the findings of Applebury et al. [2000]. We suspect that the reduced sensitivity of our indirect immunofluorescence methods as compared to those of the previous study may have prevented the reliable detection of very low levels of M-opsin expression.

In contrast to the situation in M. musculus, the number of M-opsin-expressing cones significantly exceeded the number of S-opsin-expressing cones, in both wild and laboratory stocks of $P$. maniculatus (fig. 4P-R). Indeed, density of S-cones was so low, especially in the wild Peromyscus, that it precluded a comparative analysis; at magnification levels low enough to obtain a countable number of S-cones, fluorescence was too low for reliable detection in retinal whole mounts. Therefore we did not perform dual immunocytochemical measurements. These data, of course, must be interpreted with the caveat that the antibodies used were generated specifically for the detection of Mus opsins.

\section{Discussion}

\section{Wild Mice Do Not Have Bigger Eyes}

The principal findings of this study were that the gross and cellular anatomy of the eyes of wild Mus musculus, and of wild Peromyscus maniculatus, were virtually identical to that of their respective domesticated counterparts. These findings were not anticipated, because wild mice superficially appear to have larger eyes [Austad, 2002]. Wild mice are physiologically and anatomically distinct from domesticated strains in a number of ways, and the mammalian 

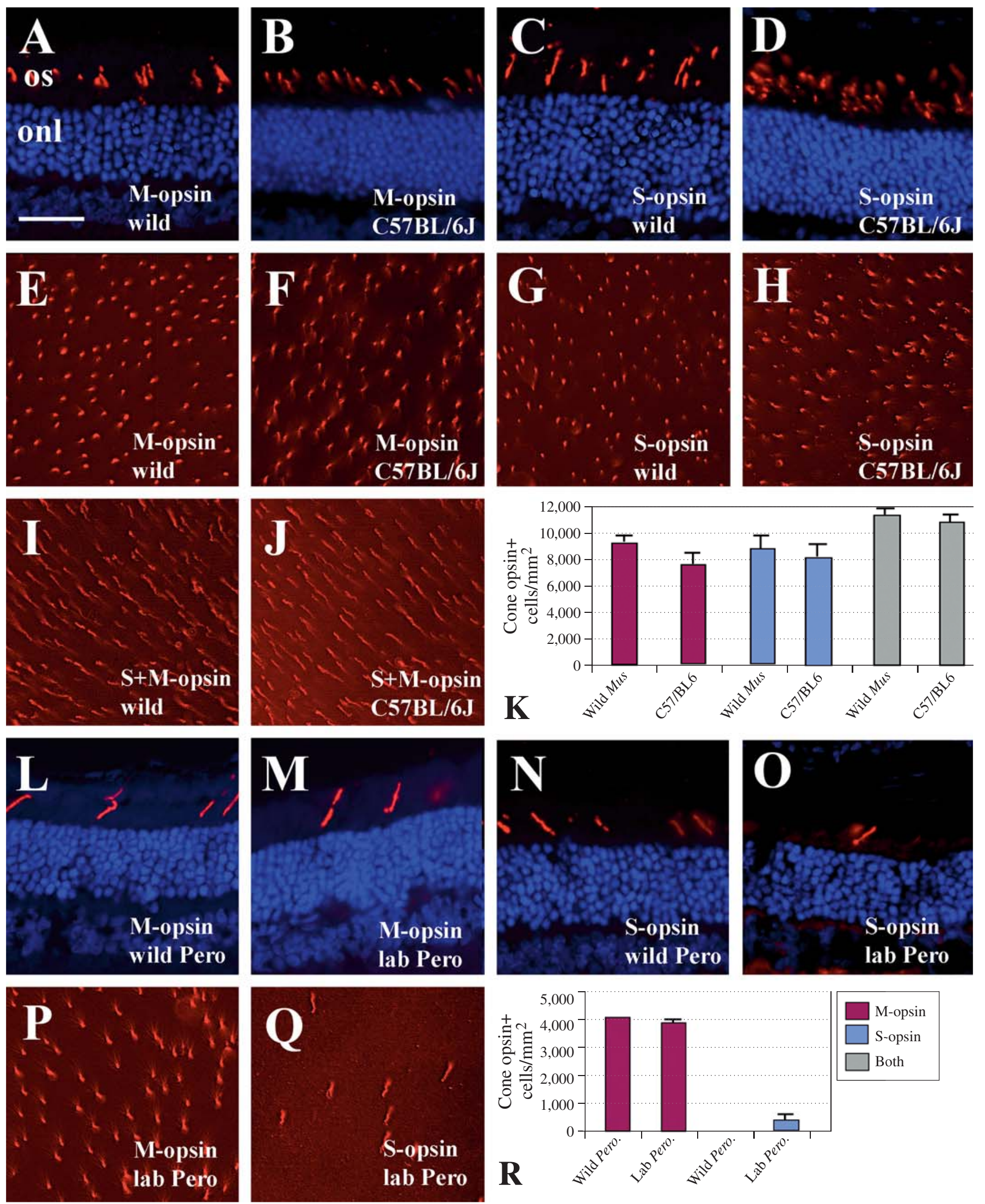
visual system is known to be susceptible to evolutionary change as a consequence of domestication [Peichl, 1992; Kruska and Sidorovich, 2003]. Nevertheless, our data demonstrate that there were no significant differences between wild-trapped $M$. musculus and $P$. maniculatus as compared to domesticated strains for the following parameters: (1) the masses of eyes and lenses; (2) the relationship between eye mass as a function of age or body mass; (3) the axial and equatorial dimensions of eyes and lenses; (4) ONL thickness, an indirect measure of photoreceptor number; (5) linear density of cells in the GCL, an indirect measure of ganglion cell number and (6) cone photoreceptor density and the densities of M- and S-cones. Our measurement and statistical methods had the power to detect differences of $15 \%$ with a $95 \%$ probability (power calculations were done using real and hypothetical means, and actual variances - mean square errors - under noncentral F-distributions), but no differences were detected. We conclude that the major features of the mouse eye that are adaptive for survival in a natural environment have been retained in mouse strains that have been under artificial selection for laboratory conditions and for experimental purposes for as long as several hundred generations.

\section{Evolutionary Effects of Domestication}

The laboratory use of $M$. musculus has resulted in selection for a number of desirable features such as rapid

Fig. 4. Cone photoreceptor densities in wild and laboratory $M$. musculus (A-K) and P. maniculatus (L-R). A-D Confirmation that cone opsin antibodies (Chemicon) specifically label outer segments of cone photoreceptors (red label) in wild M. musculus. Blue label is a DAPI nuclear counterstain; onl, outer nuclear layer; os, outer segments. Bar in A $(=50 \mu \mathrm{m})$ applies to all images in figure. A Mcone antibody; retinal cryosection from wild mouse. B M-cone antibody, C57BL/6J. C S-cone antibody, wild mouse. D S-cone antibody, C57BL/6J. E-J Retinal whole mounts labeled with anti-Mopsin, anti-S-opsin, or the combination. E M-cone antibody, wild mouse. F M-cone antibody, C57BL/6J. G S-cone antibody, wild mouse. H S-cone antibody, C57BL/6J. I Combination of M- and S-cone antibodies, wild mouse. $\mathbf{J}$ Combination of $\mathrm{M}$ - and S-cone antibodies, C57BL/6J. K Densities of cone labeling in two strains of $M$. musculus. Columns depict means plus SEM. Legend is shown in panel R. L-O Confirmation that cone opsin antibodies (Chemicon) specifically label outer segments of cone photoreceptors (red label) in wild $P$. maniculatus. L M-cone antibody; retinal cryosection from wild $P$. maniculatus. M M-cone antibody, laboratory $P$. maniculatus. N S-cone antibody, wild $P$. maniculatus. O S-cone antibody, laboratory $P$. maniculatus. P-Q Retinal whole mounts of laboratory $P$. maniculatus labeled with anti-M opsin (P) and anti-S opsin (Q). R Densities of cone labeling in two strains of $P$. maniculatus. Columns depict means \pm SEM.

The Eye of the Laboratory Mouse maturation, high fecundity, tameness, large size, and the capacity to thrive in a small cage. Laboratory selection could potentially have led to loss of alleles for highly functional eyes that would be useful under more natural conditions. Intentional inbreeding of laboratory strains to generate genetically pure lines could have also contributed to such a loss. We know this has happened for some traits. For example, one of the consequences of laboratory domestication has been a reduction in lifespan [Miller et al., 2002]. Laboratory mice also show a faster growth rate, higher fecundity [Miller et al., 2002], and most strains have lost the capacity to synthesize melatonin [Ebihara et al., 1986; Goto et al., 1989]. In addition, measures of physical fitness, such as velocity of voluntary wheel-running, $\mathrm{VO}_{2 \max }$, and size of heart ventricles, are all reduced in laboratory mice as compared to wild-derived mice [Dohm et al., 1994]. It is of interest, therefore, that basic features of the visual system do not appear to have been affected by purposeful or inadvertent laboratory selection.

We hypothesized that the visual system of laboratory strains of $M$. musculus might differ from wild $M$. musculus in ways that would influence the size of the visual field (eye size), focusing power (relationship between eye and lens size), light sensitivity and acuity (photoreceptor and ganglion cell density), and color vision (cone density and co-expression of opsins). Our rationale was that in a confined environment lacking predators with plentiful nearby food and water there would be relaxed selection pressures to maintain an optimally functional eye. This phenomenon has been demonstrated to occur in canids and mustelids. Domesticated dogs lack a high acuity area (with higher ganglion cell density) of the retina, called the visual streak, that is present in wolves [Peichl, 1992], and the numbers of retinal ganglion cells and cone photoreceptors, as well as the size of the eyes of ranched mink, are all reduced compared to wild mink [Steffen, 2000; cited in Kruska, 2005]. Furthermore, because visual structures typically carry a high metabolic cost, once they no longer serve an adaptive purpose, they can be lost or regress to the point of being nonfunctional [Cooper et al., 1993]. Classical examples include the blind mole rat [Cooper et al., 1993] and the blind cave fish [Dowling et al., 2002]. However, in these latter examples both the environment - virtual lightlessness - and the amount of time over which relaxed selection could operate are much more extreme than laboratory domestication. One might hypothesize that the seeming lack of degradation in visual acuity in laboratory mice compared with other domesticated species such as dogs is due to the much short- 
er time over which domestication has occurred [Vila et al., 1997; Savolainen et al., 2002]. However, this argument is weakened by the observation that less than 200 generations of domestication in mink has led to reduced visual acuity [Steffen, 2000; cited in Kruska, 2005]. In addition, domestication has been hypothesized to have its greatest influence on nervous systems of more highly encephalized mammals [reviewed by Kruska, 2005] than these rodents. A similar principle - less impact on less vital physiological systems -may be applicable here, such that the evolutionary effects of domestication on the visual system may be more evident and more rapid in mammals that are more dependent upon vision than are these nocturnal rodents. Canids, for example, have high acuity, stereoscopic vision and some capacity to discriminate color [Jacobs et al., 1993]. Other carnivores such as mink may require more acute vision because of their ecological role as predators [Steffen et al., 2001]. Nocturnal rodents, on the other hand, may rely less on vision and more on auditory and olfactory cues to feed and avoid predation. As a result, even assuming that selection is relaxed during domestication, the resulting changes to the rodent visual system may take many times longer than the few dozen (Peromyscus) to few hundreds (Mus) of generations over which laboratory selection has occurred thus far.

Consistent with this view, as well as with the data reported here, it is likely that the visual requirements of a wild mouse are not extensive. For instance, the refractive state of the laboratory mouse eye may be slightly hyperopic [Murphy and Howland, 1987; Schaeffel et al., 2004]. Assuming that eye size and/or axial length are predictive of refractive state [Hosny et al., 2000], our data suggest that wild mice are similarly hyperopic. The laboratory mouse eye also shows rather low acuity [approximately 0.5 cycles per degree of the visual field; Prusky et al., 2000] compared with, say, canids [11-12 cycles per degree of the visual field; Odom et al., 1983]. Assuming that ganglion cell density is predictive of visual acuity [Anderson et al., 2002], our data also suggest that wild mice have low-acuity vision. The retinas of domestic mice do have a high density of rod photoreceptors compared to primate retinas [Jeon et al., 1998]. We observed that the retinas of wild-trapped mice have statistically identical numbers of rows of ONL nuclei as those of laboratory mice. Assuming that this measure is providing information regarding rod density, this indicates that wild mice can also generate a bright retinal image, if a poorly focused one. Domesticated mice are not likely to have the capacity for true color vision, as co-expression of two opsins in the same cone photoreceptor eliminates the potential for spectral opponency [Applebury et al., 2000]. We found the same to be true for wild mice; natural populations of M. musculus are also likely to be color-blind. Eyes having high sensitivity, but low acuity, non-emmetropic refractive power, and a negligible capacity to discriminate color, must therefore be sufficient in wild mouse populations.

The environment of 'natural' populations of $M$. musculus has greatly changed over recent history, as this species has adapted to opportunistic cohabitation with humans. It is possible that humans have already played a role in the evolutionary process, and our 'wild' mice have already undergone selective changes that distinguish them from their Asian, steppe-ranging ancestors. It is therefore noteworthy that another laboratory domesticated nocturnal rodent, $P$. maniculatus, was examined. $P$. maniculatus is a new-world species that has not become as commensal as the house mouse. Our data demonstrate that, like the situation in $M$. musculus, the anatomical and histological features of the eyes of wild P. maniculatus are not significantly different from those of a domesticated laboratory strain, although admittedly Peromyscus has been subjected to laboratory domestication for a much shorter time. However, even over this short period, the laboratory $P$. maniculatus population appears to have higher densities of $\mathrm{S}$ and $\mathrm{M}$ cones than its wild relatives. However, these studies should be considered preliminary, as we examined only one or two Peromyscus retinas and only with antibodies designed for Mus cone photoreceptors.

It is of course possible that laboratory domestication resulted in evolutionary effects on the mouse visual system that we did not measure. For example, our experiments did not evaluate cellular topography within the retina and ganglion cell topography was the major difference between wolf retinas and those of domestic dogs [Peichl, 1992]. We also did not evaluate extraocular features that may have led to the impression of wild mice having larger eyes [Austad, 2002]. These features include the size of the orbit, the position, size, and contractile activity of extraocular muscles, and perhaps even the growth pattern of hair surrounding the eye. Importantly, behavioral assessments of visual function have not been done, and would be necessary to fully verify what has been inferred from these anatomical and histological studies. These experiments would clearly be worth pursuing.

The distinctive status of the laboratory mouse as one of the most carefully monitored genetic systems in biology might also explain our findings. Major heritable changes to the visual system of mice, such as photoreceptor degen- 
eration or microphthalmia, would likely be identified and selected for further study. More minor changes may occur rarely - perhaps evolutionarily regressive changes in vision happen primarily as a result of major genetic effects and not in a gradual manner. Progressive selective pressures, such as those favoring the loss of a metabolically costly, but adaptively insignificant tissue, might not be present because the metabolic costs of this tissue are of little consequence in an ad lib feeding environment or may occur at such a rate that a century of domestication is not sufficient time for it to be apparent.

\section{Validation of Mouse Models of the Visual System}

Our study provides validation for the ongoing use of the mouse model for the study of visual system structure, function, and disease. The laboratory mouse has served as a powerful genetic model for understanding disorders that influence eye development, and those that lead to photoreceptor degeneration. Some specific domesticated mouse strains are useful precisely because of genetic defects that affect the visual system [Chang et al., 2002]. The rate of accumulation of deleterious single gene mutations that affect the eye of domesticated mice has therefore been an advantage of using this inbred animal model.

The naturally-adapted mouse eye may also show agerelated anatomical changes similar to those of laboratory mice. In laboratory mice, age is associated with increased eye and lens size [Zhou and Williams, 1999], a slight decrease in depth of the vitreal chamber [Schmucker and Schaeffel, 2004], and a gradual loss of photoreceptors and ganglion cells [Gao and Hollyfield, 1992; Kunert et al., 1999; Obin et al., 2000]. Although the wild mice of known age that we evaluated were all known or assumed to be fairly young, the anatomical and histological data from these mice were consistent with the age-related trends observed for laboratory mouse strains in the present study, and with previous work by others. The implication is that the age-related changes documented for the mouse visual system are not likely the consequence of evolutionary effects of domestication. The laboratory mouse therefore remains a reasonable model for age-related visual disorders.

However, there are caveats as well as advantages to the use of the highly inbred, domesticate mouse for the pursuit of vision science. Several strains, including the C57BL/6J strain (evaluated in the present study), show a limited capacity to synthesize melatonin [Roseboom et al., 1998], a substance produced in the retina as well as in the pineal in most mammals [Tosini and Fukuhara, 2003]. The FVB strain (not evaluated in the present study), used frequently for the generation of transgenic lines, carries the $r d$ allele, which results in rapid photoreceptor degeneration [Gimenez and Montoliu, 2001]. Furthermore, some features of the mouse eye, such as a negligible capacity for color vision, make it a poor model for certain human visual functions and disorders. Concerns have been noted as to the appropriateness of the laboratory mouse eye as a model for understanding the visual systems of diurnal species that depend more explicitly on refined visual information [Schaeffel et al., 2004]. Thus, it is noteworthy to report that the basic anatomical characteristics of the eye of the laboratory mouse are consistent with the support of a visual system that is appropriately adapted to natural conditions.

\section{Acknowledgments}

The authors thank Dr. Robert Williams and Dr. Barrie Robison for critical evaluation of the manuscript, and Dr. Kirk Steinhorst for assistance with statistics. This work was supported by NIH Grants AG13711 (S.N.A.) and EY12146 (D.L.S.).

Dr. Austad's current address: University of Texas Health Sciences Center, Department of Cellular and Structural Biology, San Antonio, TX 78245-3207, USA; Dr. Kristan's current address: Departement of Biological Sciences, California State University, San Marcos, CA 92096, USA.

\section{References}

\footnotetext{
-Anderson RS, Zlatkova MB, Demirel S (2002) What limits detection and resolution of shortwavelength sinusoidal gratings across the retina? Vision Res 42:981-990.

-Applebury ML, Antoch MP, Baxter LC, Chun LL, Falk JD, Farhangfar F, Kage K, Krzystolik MG, Lyass LA, Robbins JT (2000) The murine cone photoreceptor: a single cone type expresses both $\mathrm{S}$ and $\mathrm{M}$ opsins with retinal spatial patterning. Neuron 27:513-523.

-Augustsson H, Meyerson BJ (2004) Exploration and risk assessment: a comparative study of male house mice (Mus musculus musculus) and two laboratory strains. Physiol Behav 81: 685-698.

Austad SN (2002) A mouse's tale. Nat Hist 111 : 64-70.

-Chang B, Hawes NL, Hurd RE, Davisson MT, Nusinowitz S, Heckenlively JR (2002) Retinal degeneration mutants in the mouse. Vision Res 42:517-525.

Cooper HM, Herbin M, Nevo E (1993) Visual system of a naturally microphthalmic mammal: the blind mole rat, Spalax ehrenbergi. J Comp Neurol 328:313-350.

Danias J, Lee KC, Zamora MF, Chen B, Shen F, Filippopoulos T, Su Y, Goldblum D, Podos SM, Mittag T (2003) Quantitative analysis of retinal ganglion cell (RGC) loss in aging DBA/ 2Nnia glaucomatous mice: comparison with RGC loss in aging C57/BL6 mice. Invest Ophthalmol Vis Sci 44:5151-5162.
} 
Dohm MR, Richardson CS, Garland T Jr (1994) Exercise physiology of wild and random-bred laboratory house mice and their reciprocal hybrids. Am J Physiol 267:R1098-R1108.

- Dowling TE, Martasian DP, Jeffery WR (2002) Evidence for multiple genetic forms with similar eyeless phenotypes in the blind cavefish, Astyanax mexicanus. Mol Biol Evol 19:446455.

Dräger UC, Olsen JF (1981) Ganglion cell distribution in the retina of the mouse. Invest Ophthalmol Vis Sci 20:285-293.

-Ebihara S, Marks T, Hudson DJ, Menaker M (1986) Genetic control of melatonin synthesis in the pineal gland of the mouse. Science 231: 491-493.

- Farber DB, Tsang SH (2003) Stationary night blindness or progressive retinal degeneration in mice carrying different alleles of PDE gamma. Front Biosci 8:s666-s675.

-Fernandez C, Liu L, Paya-Cano JL, Gregorova S, Forejt J, Schalkwyk LC (2004) Behavioral characterization of wild derived male mice (Mus musculus musculus) of the PWD/Ph inbred strain: high exploration compared to C57BL/6J. Behav Genet 34:621-630.

-Frederick JM, Krasnoperova NV, Hoffmann K, Church-Kopish J, Ruther K, Howes K, Lem J, Baehr W (2001) Mutant rhodopsin transgene expression on a null background. Invest Ophthalmol Vis Sci 42:826-833.

-Frick H, Nord HJ (1963) Domestikation und Hirngewicht. Anat Anz 113:307-316.

Gao H, Hollyfield JG (1992) Aging of the human retina. Differential loss of neurons and retinal pigment epithelial cells. Invest Ophthalmol Vis Sci 33:1-17.

- Gimenez E, Montoliu L (2001) A simple polymerase chain reaction assay for genotyping the retinal degeneration mutation $(\operatorname{Pdeb}(\mathrm{rd} 1))$ in FVB/N-derived transgenic mice. Lab Anim 35: 153-156.

Goto M, Oshima I, Tomita T, Ebihara S (1989) Melatonin content of the pineal gland in different mouse strains. J Pineal Res 7:195-204.

-Hemann MT, Greider CW (2000) Wild-derived inbred mouse strains have short telomeres. Nucl Acids Res 28:4474-4478.

- Hosny M, Alio JL, Claramonte P, Attia WH, PerezSantonja JJ (2000) Relationship between anterior chamber depth, refractive state, corneal diameter, and axial length. J Refract Surg 16: 336-340.
Jacobs GH, Deegan JF II, Crognale MA, Fenwick JA (1993) Photopigments of dogs and foxes and their implications for canid vision. Vis Neurosci 10:173-180.

Jeon CJ, Strettoi E, Masland RH (1998) The major cell populations of the mouse retina. J Neurosci 18:8936-8946.

Kruska DCT (1996) The effect of domestication on brain size and composition in the mink (Mustela vison). J Zool Lond 239:645-661.

Kruska DCT (2005) On the evolutionary significance of encephalization in some eutherian mammals: effects of adaptive radiation, domestication, and feralization. Brain Behav Evol 65:73-108.

Kruska DCT, Sidorovich VE (2003) Comparative allometric skull morphometrics in mink (Mustela vison Schreber, 1777) of Canadian and Belarus origin; taxonomic status. Mamm Biol 68: 257-276.

Kunert KS, Fitzgerald ME, Thomson L, Dorey CK (1999) Microglia increase as photoreceptors decrease in the aging avian retina. Curr Eye Res 18:440-447.

Miller RA, Harper JM, Dysko RC, Durkee SJ, Austad SN (2002) Longer life spans and delayed maturation in wild-derived mice. Exp Biol Med (Maywood) 227:500-508.

Murphy CJ, Howland HC (1987) The optics of comparative ophthalmoscopy. Vision Res 27: 599-607.

Obin M, Pike A, Halbleib M, Lipman R, Taylor A, Bronson R (2000) Calorie restriction modulates age-dependent changes in the retinas of Brown Norway rats. Mech Ageing Dev 114: 133-147.

Odom JV, Bromberg NM, Dawson WW (1983) Canine visual acuity: retinal and cortical field potentials evoked by pattern stimulation. Am J Physiol 245:R637-R641.

Peichl L (1992) Topography of ganglion cells in the dog and wolf retina. J Comp Neurol 324:603620 .

Prusky GT, West PW, Douglas RM (2000) Behavioral assessment of visual acuity in mice and rats. Vision Res 40:2201-2209.

Roseboom PH, Namboodiri MA, Zimonjic DB, Popescu NC, Rodriquez IR, Gastel JA, Klein DC (1998) Natural melatonin 'knockdown' in C57BL/6J mice: rare mechanism truncates serotonin $\mathrm{N}$-acetyltransferase. Brain Res Mol Brain Res 63:189-197.
Savolainen P, Zhang YP, Luo J, Lundeberg J, Leitner $T$ (2002) Genetic evidence for an East Asian origin of domestic dogs. Science 298: 1610-1613.

Schaeffel F, Burkhardt E, Howland HC, Williams RW (2004) Measurement of refractive state and deprivation myopia in two strains of mice. Optom Vis Sci 81:99-110.

Schmucker C, Schaeffel F (2004) A paraxial schematic eye model for the growing C57BL/6 mouse. Vision Res 44:1857-1867.

Silver LE (1995) Mouse Genetics: Concepts and Applications. Oxford, UK: Oxford University Press.

-Smith SB (1992) C57BL/6J-vit/vit mouse model of retinal degeneration: light microscopic analysis and evaluation of rhodopsin levels. Exp Eye Res 55:903-910.

Steffen K (2000) Vergleichender quantitativer Nachweis sowie topographische Analyse von Ganglienzellen und Zapfen in der Retina von Wildmink (Mustela vison energumenos) und Farmmink (Mustela vison $\mathrm{f}$. dom.). Diss. thesis, Math-Naturwiss. Fak., Universität Kiel.

Steffen K, Kruska D, Tiedemann R (2001) Postnatal brain size decrease, visual performance, learning, and discrimination ability of juvenile and adult American mink (Mustela vison: Carnivora: Mammalia). Mamm Biol 66:269280.

$>$ Stenkamp DL, Frey RA (2003) Extraretinal and retinal hedgehog signaling sequentially regulate retinal differentiation in zebrafish. Dev Biol 258:349-363.

Stenkamp DL, Frey RA, Prabhudesai SN, Raymond PA (2000) Function for Hedgehog genes in zebrafish retinal development. Dev Biol 220:238-252.

Tosini G, Fukuhara C (2003) Photic and circadian regulation of retinal melatonin in mammals. $J$ Neuroendocrinol 15:364-369.

Vila C, Savolainen P, Maldonado JE, Amorim IR, Rice JE, Honeycutt RL, Crandall KA, Lundeberg J, Wayne RK (1997) Multiple and ancient origins of the domestic dog. Science 276:16871689.

Zheng QY, Johnson KR, Erway LC (1999) Assessment of hearing in 80 inbred strains of mice by ABR threshold analyses. Hearing Res 130:94107.

Zhou G, Williams RW (1999) Mouse models for the analysis of myopia: an analysis of variation in eye size of adult mice. Optom Vis Sci 76: 408-418. 